\title{
Correction to: Treatment of adults with intracranial hemorrhage on apixaban or rivaroxaban with prothrombin complex concentrate products
}

\author{
Renee Castillo ${ }^{1} \cdot$ Alissa Chan $^{3} \cdot$ Steven Atallah ${ }^{1} \cdot$ Katrina Derry $^{3} \cdot$ Mark Baje $^{1} \cdot$ Lara L. Zimmermann $^{6} \cdot$ Ryan Martin $^{6}$. \\ Leonid Groysman ${ }^{2}$. Sara Stern-Nezer ${ }^{2}$. Anush Minokadeh ${ }^{4}$. Alan Nova ${ }^{4} \cdot$ WanTing Huang $^{3} \cdot$ William Cang ${ }^{3}$. \\ Kendra Schomer ${ }^{5}$
}

Published online: 23 June 2020

(c) Springer Science+Business Media, LLC, part of Springer Nature 2020

\section{Correction to: Journal of Thrombosis and Thrombolysis https://doi.org/10.1007/s11239-020-02154-z}

In the original publication of the article, unfortunately the given name and family name of the author's in the author group were inadvertently interchanged. This has been corrected with this erratum article.
Publisher's Note Springer Nature remains neutral with regard to jurisdictional claims in published maps and institutional affiliations.

The original article has been corrected.

The original article can be found online at https://doi.org/10.1007/ s11239-020-02154-z.

\section{Kendra Schomer}

kjschomer@ucdavis.edu

1 Department of Pharmacy, University of California, Irvine Health, Orange, CA, USA

2 Department of Neurology, University of California, Irvine Health, Orange, CA, USA

3 Department of Pharmacy, University of California San Diego Health, San Diego, CA, USA

4 Department of Critical Care, University of California San Diego Health, San Diego, CA, USA

5 Department of Pharmacy, University of California Davis Health, Sacramento, CA, USA

6 Department of Neurological Surgery and Neurology, University of California Davis Health, Sacramento, CA, USA 